\title{
Dysplastic bone disease mimicking exostoses of the ear canal
}

A Shayah, MRCS, DOHNS
C Gadepalli, MRCS
D Loke, FRCS
L C Knight, MPhil, FRCS
Department of Otolaryngology - Head and Neck Surgery, Leeds General
Infirmary, Leeds, UK

\section{Abstract}

We present a case of a 43-year-old man who attended the ENT clinic complaining of bilateral hearing loss. He had multiple bony swellings in both ear canals that mimicked exostoses. An audiogram showed bilateral symmetrical mixed hearing loss. Excision was carried out to facilitate the use of a hearing aid. Intraoperatively, the swellings were confluent, extending down to the annulus. The blood tests and skull X-ray were suggestive of osteopetrosis. Following surgery, the patient was referred to the medical team.

\section{Introduction}

Patients with systemic bone disease presenting to the ENT department are rare. We report an interesting case of a patient who presented with gradual onset of deafness and external auditory canal swellings, mistaken initially for multiple simple exostoses of both ear canals, in combination with mild background sensorineural hearing loss.

\section{Case report}

The patient was a 43-year-old man who was referred to the ENT department complaining of gradual deterioration in hearing in both ears. He had a history of being an avid swimmer. There were no other otological symptoms. On examination, he had multiple bony swellings in both ear canals. The tympanic membranes were normal. A pure tone audiogram showed bilateral symmetrical mixed hearing loss.

A provisional diagnosis of exostoses with sensorineural hearing loss was made. The patient was initially prescribed a hearing aid. Unfortunately, the hearing aid did not function well due to the poor fitting of ear moulds in the narrow ear canal. Excision of the osteomas and canaloplasty was therefore undertaken on the narrowest canal to facilitate hearing aid effectiveness.

Intra-operatively, these bony swellings were found to be a solid bony mass under the meatal skin, extending down to the level of the annulus. The ossicles appeared normal and mobile. The excess bone was drilled out and a temporalis fascia graft was used to close a small iatrogenic perforation of the posterior tympanic membrane. The appearance of these thickened bony swellings was not typical of exostoses.

In retrospect, the patient was observed to have an enlarged cranium. Further tests were conducted to investigate metabolic or dysplastic bone disease. It also transpired, on direct questioning, that the patient had a maternal history of osteopetrosis. The liver function tests showed

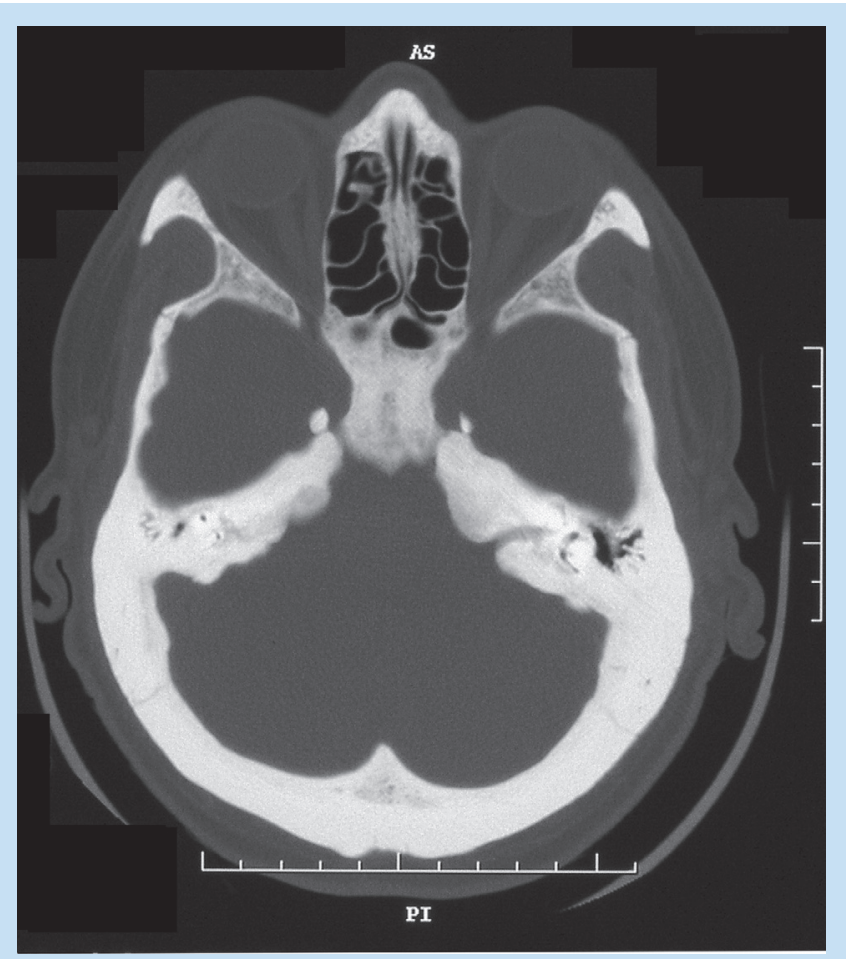

Fig. 1. Axial CT scan showing generalised thickening of the cranial vault.

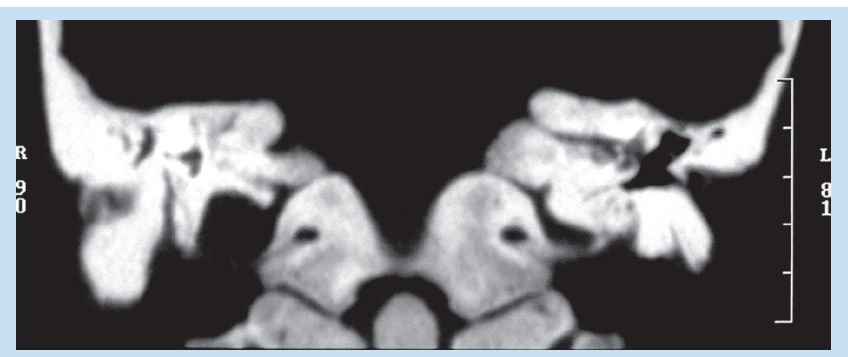

Fig. 2. Temporal bone CT scan showing narrowing in both internal auditory canals and constriction of the contents of both otic capsules.

raised serum alkaline phosphatase; however, serum calcium, full blood count, urea and electrolytes were all normal. Imaging studies of the skull (Fig. 1) and lumbar and thoracic spine radiography showed generalised thickening and bony sclerosis in keeping with the diagnosis of osteopetrosis.

A temporal bone computed tomography (CT) scan revealed narrowing in both internal auditory canals and constriction of the contents of both otic capsules. All these features were suggestive of osteopetrosis (Fig. 2), with the exception of the raised alkaline phosphate which is more typical of Paget's disease than of osteopetrosis. However, the patient was reviewed by the endocrinology team who thought that the elevated alkaline phosphate was attributed to high alcohol consumption and obesity. Therefore, the final diagnosis of osteopetrosis was made on the clinical and imaging features. No treatment was recommended apart 
from lifestyle changes and reducing his alcohol intake. Further genetic study was planned in view of his family history.

\section{Discussion}

Various metabolic and dysplastic diseases can involve the temporal bone but rarely present initially to the ENT clinic. We report the case of a man who presented with bilateral hearing impairment thought to be related to ear canal exostosis. Family history and imaging studies guided the diagnosis toward osteopetrosis, a sclerotic dysplastic disorder of bone.

Osteopetrosis is a heterogeneous disorder characterised by the failure of osteoclasts to resorb bone. As a consequence, bone modelling and remodelling are impaired. Osteopetrosis was first described by a German radiologist, Albers-Schönberg, in $1904 .^{1}$ The estimated global incidence is up to 1:500 $000{ }^{2}$ The defect in bone turnover characteristically results in skeletal fragility despite increased bone mass, and it may also cause haematopoietic insufficiency, disturbed tooth eruption, nerve entrapment syndromes, and growth impairment.

Deafness is usually conductive, secondary to anomalous bone formation ${ }^{3}$ or tubal blockade. ${ }^{4}$ Sensorineural hearing loss secondary to nerve compression in the internal auditory meatus has been reported. ${ }^{3}$

There are three clinical types of osteopetrosis: 'malignant' (infantile), 'benign' (adult) and intermediate. The so-called 'malignant' form is an autosomal recessive disease and presents in infants and children as anaemia and neutropenia, neuropathy secondary to direct compression of nerve or resulting from vascular infarction, severe sensorineural hearing loss, mental retardation, hydrocephalus, multiple fractures and hypocalcaemia. This form has a very poor prognosis. ${ }^{5}$

The 'benign' form is autosomal dominant, is also called AlbersSchönberg disease, and affects adults. This form can be asymptomatic or present with macrocephaly, widening of the mandible, proptosis, conductive hearing loss and sensorineural hearing loss. Narrowing of the internal and external acoustic meatus (sensorineural loss) and sclerosis of the skull may occur. ${ }^{5}$

The intermediate autosomal recessive form appears during the first decade of life and has features in common with the benign form. ${ }^{6}$

Two genes have been shown to be associated with osteopetrosis: Atp6a3 (TCIRG1) associated with type 1 (autosomal recessive) and ClCN7 associated with type 2 (autosomal dominant). However, the genetic basis of type 3 (intermediate) is unknown.

Treatment of the benign form is entirely symptomatic, aimed at correcting facial deformity or recurrent fractures. Surgery may also be indicated to treat neuropathy related to nerve compression. ${ }^{8}$ However, in the infantile malignant form, studies have shown that a bone marrow transplant is the only curative treatment. ${ }^{8}$ Other methods of treatment for the malignant disease have included gamma interferon and calcitrol (1,25-dihydroxy vitamin D). ${ }^{8}$

Skull thickening can be encountered in various other conditions. Chronic phenytoin therapy has shown to provoke osteoblast proliferation, causing skull thickening which can develop in $34 \%$ of patients with seizure disorders on long-term phenytoin therapy. The consequences of skull thickening are mainly cosmetic, giving the features of acromegaly with normal size of hands and feet. ${ }^{9}$

Similar features can also present in Van Buchem disease, which was first described in 1955 as an autosomal recessive bone dysplasia linked to a genetic locus on chromosome 17q12-21. This disease is characterised by asymmetrically increased thickness of bones (mostly jawbone) but can also present in skull, ribs, long bones as well as hands and feet, resulting in increased cortical bone density. This may lead to facial nerve palsy causing hearing loss, visual problems, neurological pain and, very rarely, blindness as a consequence of optic atrophy. Bone anomalies appear in the first decade of life and become more prominent among the elderly. ${ }^{10}$

Other causes of bilateral hyperostosis of the skull vault are marrow hyperplasia, hyperparathyroidism and acromegaly. None of these conditions was evident in the in the above case.

1. Waguespack SG, Hui SL, White KE, Buckwalter KA, Econs MJ. Measurement of tartrate-resistant acid phosphatase and the brain isoenzyme of creatine kinase accurately diagnoses type II autosomal dominant osteopetrosis but does not identify gene carriers. J Clin Endocrinol Metab 2002; 87(5): 2212-2217.

2. Beighton P, Hamersma H, Cremin BJ. Osteopetrosis in South Africa. The benign, lethal and intermediate forms. S Afr Med J 1979; 55(17): 659-665.

3. Milroy CM, Michaels L. Temporal bone pathology of adult-type osteopetrosis. Arch Otolaryngol Head Neck Surg 1990; 116(1): 79-84

4. Magliulo G, Celebrini A, Cuiuli G, Parotto D. Osteopetrosis of temporal bone with blockage of the eustachian tube. A case report. An Otorrinolaringol Ibero Am 2004; 31(1): 51-58.

5. Antunes ML, Testa JR, Frazatto R, Barberi JA, Silva RF. Rare osteodysplasia of the temporal bone. Rev Bra Otorrinolaringol 2005; 71(2): 228-232.

6. Frederic S. Osteopetrosis: Current clinical considerations. Clin Orthop 1993; 294: 34-44.

7. Frattini A, Pangrazio A, Susani L, et al. Chloride channel ClCN7 mutations are responsible for severe recessive, dominant, and intermediate osteopetrosis. J Bone Miner Res 2003; 18(10): 1740-1747.

8. Carolino J, Perez JA, Popa A. Osteopetrosis. Am Fam Physician 1998; 57(6): 1293-1296.

9. Chow KM, Szeto CC. Cerebral atrophy and skull thickening due to chronic phenytoin therapy. CMAJ 2007; 176(3): 321-323.

10. Wergedal JE, Veskovic K, Hellan M, et al. Patients with Van Buchem disease, an osteosclerotic genetic disease, have elevated bone formation markers, higher bone density, and greater derived polar moment of inertia than normal. J Clin Endocrinol Metab 2003; 12: 5778-5783. 